\title{
From keratinocyte to cancer: the pathogenesis and modeling of cutaneous squamous cell carcinoma
}

\author{
Vladimir Ratushny, Michael D. Gober, Ryan Hick, Todd W. Ridky, and John T. Seykora
}

Department of Dermatology, Perelman School of Medicine, University of Pennsylvania, Philadelphia, Pennsylvania, USA.

\begin{abstract}
Cutaneous squamous cell carcinoma (cSCC) is the second most common human cancer with over 250,000 new cases annually in the US and is second in incidence only to basal cell carcinoma. cSCC typically manifests as a spectrum of progressively advanced malignancies, ranging from a precursor actinic keratosis (AK) to squamous cell carcinoma (SCC) in situ (SCCIS), invasive cSCC, and finally metastatic SCC. In this Review we discuss clinical and molecular parameters used to define this range of cutaneous neoplasia and integrate these with the multiple experimental approaches used to study this disease. Insights gained from modeling cSCCs have suggested innovative therapeutic targets for treating these lesions.
\end{abstract}

\section{Introduction}

Actinic keratoses (AKs) are likely the most common keratinocytederived precancerous lesion in humans; they are found predominantly in fair-skinned individuals on sun-exposed surfaces $(1,2)$. The primary risk factor for AKs is cumulative UV exposure from sunlight and/or tanning salons, and it is expected that the incidence of AKs will rise given an aging US population (3-5). AKs are frequently found in skin harboring fully developed cutaneous squamous cell carcinomas (cSCCs) (6). AKs progress to cSCC at a rate estimated between $0.025 \%$ and $16 \%$ for an individual lesion per year $(7,8)$. The typical patient has 6 to 8 lesions; therefore, a patient with multiple AKs has a annual risk of developing invasive squamous cell carcinoma (SCC) ranging from $0.15 \%$ to $80 \%$ $(2,3,6,7)$. This wide range in risk reflects a lack of precision in our knowledge of the progression of carcinoma in the epidermis. It is estimated that approximately $26 \%$ of AKs will undergo regression over a year's span (8). The probability of an AK or a patient with multiple AKs developing a cSCC or metastatic lesion is shown in Figure $1(9,10)$.

AKs are defined at the histologic level by dysplasia and consist of keratinocytes manifesting atypical nuclei that are enlarged, irregular, and hyperchromatic. AKs also display disorganized growth, which disrupts differentiation and results in a thickened stratum corneum with retained nuclei. To stratify degrees of epidermal dysplasia, a three-tiered grading scale has been proposed for AKs that parallels that used for evaluation of cervical dysplasia (11). The histological features of keratinocytic intraepidermal neoplasia I (KIN I) are cellular atypia of basal keratinocytes confined to the lower third of the epidermis. KIN II shows atypical keratinocytes occupying the lower two-thirds of the epidermis, and KIN III shows atypical keratinocytes throughout the epidermis; this latter stage is equivalent to carcinoma in situ (11). The localized epidermal atypia in AKs reflects a partial disruption of the differentiation program, whereas a more complete disruption of differentiation is associated with SCC in situ (SCCIS). The regression rate of AKs may be inversely related to their degree of dysplasia, as has been seen in vaginal epithelium (12). While the KIN grad-

Conflict of interest: The authors have declared that no conflict of interest exists. Citation for this article: J Clin Invest. 2012;122(2):464-472. doi:10.1172/JCI57415. ing criteria evaluate the macroscopic and microscopic features of AKs, identification of genetic and molecular abnormalities associated with these lesions has provided mechanistic insight into their pathogenesis (Figure 2).

The classic multistep model of carcinogenesis is useful for understanding the progression from AK to cSCC (13). According to this model, mutations in one gene, often a tumor suppressor, may lead to the development of a precursor lesion with increased genetic instability or loss of cell cycle control. Additional mutations in other driver oncogenes permit the emergence of more neoplastic properties, leading to invasive carcinoma; the number of genetic changes required to transition from benign epithelium to metastatic carcinoma internal malignancies is thought to range from four to six (13). However, 3D models of human epidermis have shown that as few as two proto-oncogene mutations changes are sufficient to drive SCC $(14,15)$. An improved understanding of epigenetic regulation of oncogene and anti-oncogene expression will add layers of regulatory complexity to the multistep model of carcinogenesis.

As with other cancers, cSCC exhibits impaired genomic maintenance that facilitates acquisition of new mutations (16). The mechanism leading to genomic instability in keratinocytes likely results from UVB-induced inactivation of p53, since approximately $58 \%$ of cSCCs harbor UVB signature mutations such as $\mathrm{CC} \rightarrow \mathrm{TT}$ and $\mathrm{C} \rightarrow \mathrm{T}$ transitions (17). The role of $\mathrm{p} 53$ in UVBinduced carcinogenesis has been confirmed in $p 53^{-/-}$mice which have an increased propensity for developing AK-like lesions and cSCCs secondary to UVB exposure (18). Since the initial observations of ultraviolet-induced mutations in $p 53$, other groups have confirmed the presence of $\mathrm{p} 53$ mutations in significant percentages of cSCCs (19-21).

p53 is mutated commonly in AKs, demonstrating that dysplastic lesions have acquired the initial genetic mutations prior to becoming $\operatorname{cSCC}(20,22)$. Additional studies have shown a high prevalence $(74 \%)$ of $p 53$ mutations in unremarkable sun-exposed skin compared to non-sun-exposed skin (5\%) (23), setting the stage for acquisition of mutations in driver oncogenes. Consistent with these findings, $40 \%$ of SCCIS harbors p53 mutations, indicating that p53 loss occurred prior to tumor invasion (24). In contrast, several internal malignancies demonstrate mutation of the $p 53$ 


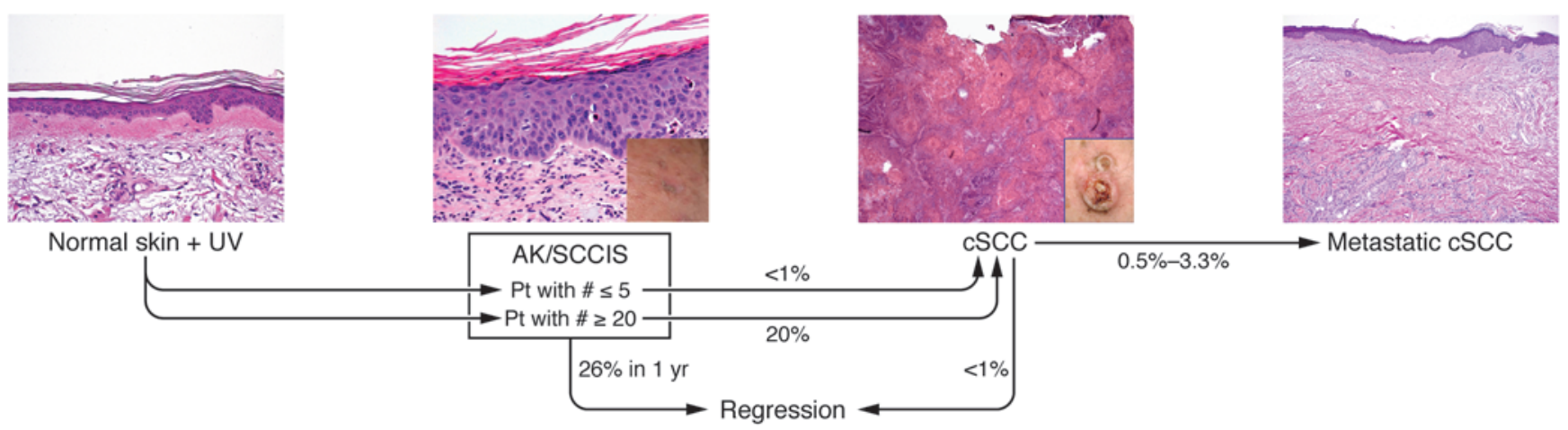

Figure 1

Probability that human cutaneous neoplastic lesions will progress to invasive carcinoma. The likelihood of AKs in patients with fewer than 5 or more than 20 lesions progressing to cSCC is shown $(9,10)$.

gene as a late event in neoplastic evolution after formation of an invasive lesion (25-27).

Aberrant activation of EGFR and Fyn, a Src-family tyrosine kinase (SFK), are seen in human cSCCs, and these kinases downregulate p53 mRNA and protein levels through a c-Jun-dependent mechanism $(28,29)$, revealing another mechanism for controlling p53 function. Additional molecular events associated with AK formation include increased activation or levels of SFKs, EGFR, Myc, and ATF-3 (30-33). In addition, decreased levels of inositol polyphosphate 5 '-phosphatase have also been reported in AKs, which could result in increased PI3K/Akt signaling (34).

Immunohistochemical studies of AKs to assess p53 levels have shown variable results, but many studies show increased p53 levels in lesional cells that are likely due to the enhanced stability of the mutant p53 proteins (35). However, more mechanistic studies are needed to link specific p53 mutations found in human AKs with altered functional status and protein stability.

Loss-of-heterozygosity studies of AKs have shown genetic alterations at the following loci: $3 \mathrm{p}, 9 \mathrm{p}, 9 \mathrm{q}, 13 \mathrm{q}, 17 \mathrm{p}$, and $17 \mathrm{q}$; these data indicate that substantial genomic instability is already present at the preneoplastic AK stage (36). However, the genes, microRNAs (miRNAs), and long non-coding RNAs (lncRNAs) affected by these genetic alterations have not yet been linked to mechanisms driving neoplasia.

Loss of heterozygosity has also been observed in cSCCs at chromosome 9p in 13 of 16 primary tumors (37). Loss of heterozygosity of $\mathrm{p} 16$, a cell cycle regulator that lies in this region, is hypothesized to be associated with progression from AKs to cSCCs (38), and loss of function of $\mathrm{p} 16$ is more frequent in cSCCs than in precancerous lesions (39-41). Additional loss-of-heterozygosity loci in cSCCs include 3 p, 2q, 8p, and 13 and allelic gains on $3 q$ and $8 \mathrm{q}(37)$.

Amplification and activating mutations of the Ras oncogene have been found in SCCs and AKs $(42,43)$. Of the three Ras genes, Harvey rat sarcoma virus oncogene (Hras) is preferentially mutated in the general population (44). Ras molecules are a family of GTPbinding proteins that are among the most frequently mutated genes in humans cancers (45). Ras is an upstream activator of the Raf/Mek/Erk1/Erk2 kinase pathway, and activating mutations in Ras can promote cSCC formation (45). The latest data from the catalog of somatic mutations in cancer (COSMIC; Sanger Institute) indicate that $21 \%$ of cSCCs harbor activating Ras mutations (9\% Hras, 7\% Nras, 5\% Kras) (46). The characteristic mutations at codons 12, 13, and 61 of $\mathrm{H}$-Ras are all localized opposite pyrimidine dimer sites (C-C) and likely result from UVB exposure (42).

\section{Role of extracellular matrix and basement membrane components in SCC}

The interactions between tumor cells with their extracellular matrix and the basement membrane zone (BMZ) are important determinants of tumorigenesis and have been shown to play a role in cSCC (47). Laminin 332 (previously known as laminin 5) is a BMZ component involved in epithelial-mesenchymal cohesion in multiple tissues including the epidermis (47). Laminin 332 and one of its ligation partners, $\alpha 6 \beta 4$ integrin, were shown to be required for tumorigenesis in a murine xenograft model of human SCC (14). In this model, tumor formation by human keratinocytes transformed through retrovirally mediated expression of HRAS and ІкB $\alpha$ was completely inhibited with blocking antibodies to laminin 332 or $\beta 4$ integrin. Collagen VII is a component of anchoring fibrils in the $\mathrm{BMZ}$, which anchor the basement membrane to the underlying dermis. In a 3D organotypic skin model of SCC, siRNA-mediated depletion of collagen VII promoted migration, invasion, disorganized differentiation, and epithelial-mesenchymal transition (48). The 3D organotypic model has been used to define gene expression profiles in the epithelial and stromal components of tumors important for carcinogenesis (49). Expression of $\beta 1$-integrins and their ligands correlates with tumor progression in human skin, so further characterization of the mitogenic signaling mechanisms between HRAS and the integrin/BMZ complex will likely increase our understanding of cSCC pathogenesis.

\section{Identifying the cell of origin in SCC}

In the hair follicle bulge and the basal layer of the interfollicular epidermis, epidermal stem cells have the potential for self-renewal and multi-lineage differentiation and have been pegged as putative cells of origin for cSCC (50). In a chemical carcinogenesis model of cSCC, 7,12-dimethyl-1,2-benzanthracene (DMBA) initiated epidermal tumor formation in mice treated with 5-fluorouracil (5-FU), a compound that kills actively cycling cells in the epidermis (51). These data suggest that the tumors arose from the quiescent, 5-FUinsensitive epidermal stem cells rather than the rapidly proliferating epidermal cells. When oncogenic Hras was expressed in suprabasal cells of the mouse epidermis under the keratin 10 gene promoter, benign papillomas, a benign squamous skin tumor seen in murine 


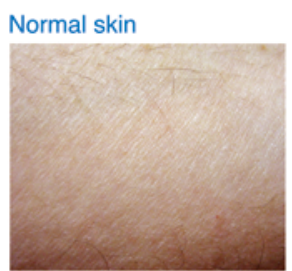

Clinical

description

Histopathology

description basalis, spinosum, and

granulosum with

orthokeratotic scale

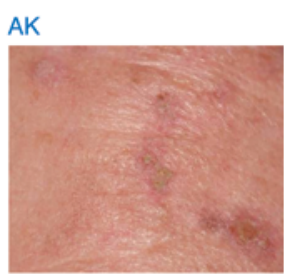

Scaly skin colored/pink macule or papule

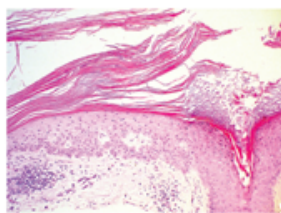

Enlarged, atypical keratinocytes confined to the epidermis with parakeratotic scale

ras

bcl-2

overexpression,

or amplification)

Decreased signaling (deactivation, transcriptional or translational repression, or gene deletion)

Genomic changes keratinocytes invading

p53

Srcasm

Genomic instability with few chromosomal alterations

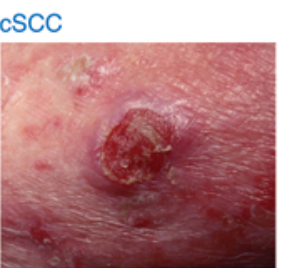

Persistent firm or scaly papule or red nodule which may spontaneously bleed

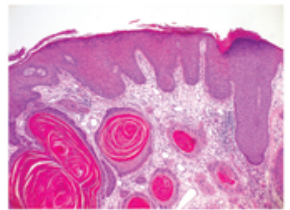

Enlarged, atypical the dermis

ras

Fyn/SFKs

c-myc

bcl-2

STAT-3

MMP

p53

Srcasm

Notch (p53)

PKC $\delta$

E-cadherin

Increased genomic instability resulting in chromosoma

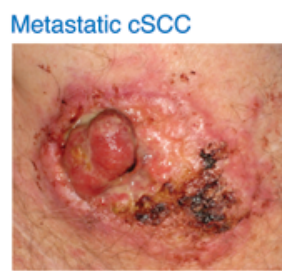

Multiple nodular lesions

in skin or internal organs

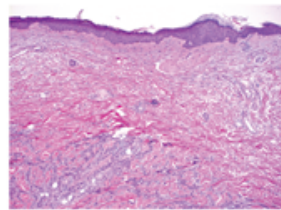

Enlarged, atypical keratinocytes in the dermis, lymph nodes, or internal organs, typically with no epidermal connection

In addition to $\mathrm{CSCC}$ alterations:

VEGF (ras)

MMP2

MMP7

MMP12 (ras)

In addition to CSCC alterations:

E-cadherin

P-cadherin

translocations, isochromosomes, gene deletions, and amplifications

\section{Figure 2}

A clinical, histologic, and molecular comparison of AKs, cSCC, and metastatic cSCC. The common clinical, histologic, and molecular features of human cutaneous neoplasia are shown.

models, arose at sites of wounding (52). In contrast, when this same oncogene was expressed in the hair follicle under the control of the truncated keratin 5 gene promoter, cSCC and some spindle cell carcinomas developed in adult mice (53). Another approach to identify the cSCC cell of origin involved expressing a constitutively active mutant form of KRAS (G12D) at physiological levels in multiple epidermal compartments using a tamoxifen-inducible CRE-Lox system (54). The expression of mutated KRAS in hair follicle bulge stem cells, but not in the developmentally committed transient amplifying matrix cells, resulted in formation of papillomas. Although KRAS $^{\mathrm{G} 12 \mathrm{D}}$ expression alone was not sufficient to induce malignant transformation, the combination of this oncogene with a deletion of 553 in bulge stem cells induced invasive SCC, but the combination was not tumorigenic in transient amplifying matrix cells. There is also evidence that bone marrow-derived cells may home to the bulge region of the epidermis in response to skin wounding and differentiate into keratinocyte stem cells (55). These bone marrowderived cells possess the capacity to undergo malignant transformation with persistent stimulation (i.e., chronic inflammation) in their new environment $(50,56,57)$. Considering the differences in epidermal stem cells and the determinants of tumorigenesis between mice and humans, further investigation into the role of cancer propagating cells in human cSCC is warranted.
Pathophysiologic features of cSCCs. Ras family members of proto-oncogenes transduce cellular growth and proliferation signals downstream of cell membrane-bound receptor tyrosine kinases (RTKs). Ras can be activated by gene amplification, activating mutations, or overexpression of upstream RTKs. Aberrant Ras activation promotes several key tumorigenic phenotypes including mitogenesis, resistance to apoptosis, drug resistance, and angiogenesis (45).

It was experimentally shown that activation of oncogenic Ras alone was not sufficient to induce SCC in human keratinocytes (58). Instead, it was necessary to couple Ras overexpression with the activation of the cell cycle progression mediator CDK4, or to modulate NF- $\kappa \mathrm{B}$ activity to bypass Ras-mediated G1 arrest and induce epidermal tumorigenesis (14). NF- $\kappa \mathrm{B}$ is important for growth inhibition in keratinocytes, and blockade of this pathway induces cSCC in murine experimental models $(59,60)$. Epidermal hyperproliferation secondary to NF-кB blockade was associated with increased CDK4 protein levels, thus linking control of the G1/S cell cycle transition to the growth regulatory functions of NF-кB (59). These experimentally produced tumors mimicked human cSCCs by demonstrating E-cadherin downregulation and induction of angiogenic and invasive cellular factors $(14,15,45)$.

One recent observation in a human keratinocyte cell line $\mathrm{HaCaT}$ cells) demonstrated that the Fyn kinase is an effector of oncogenic 


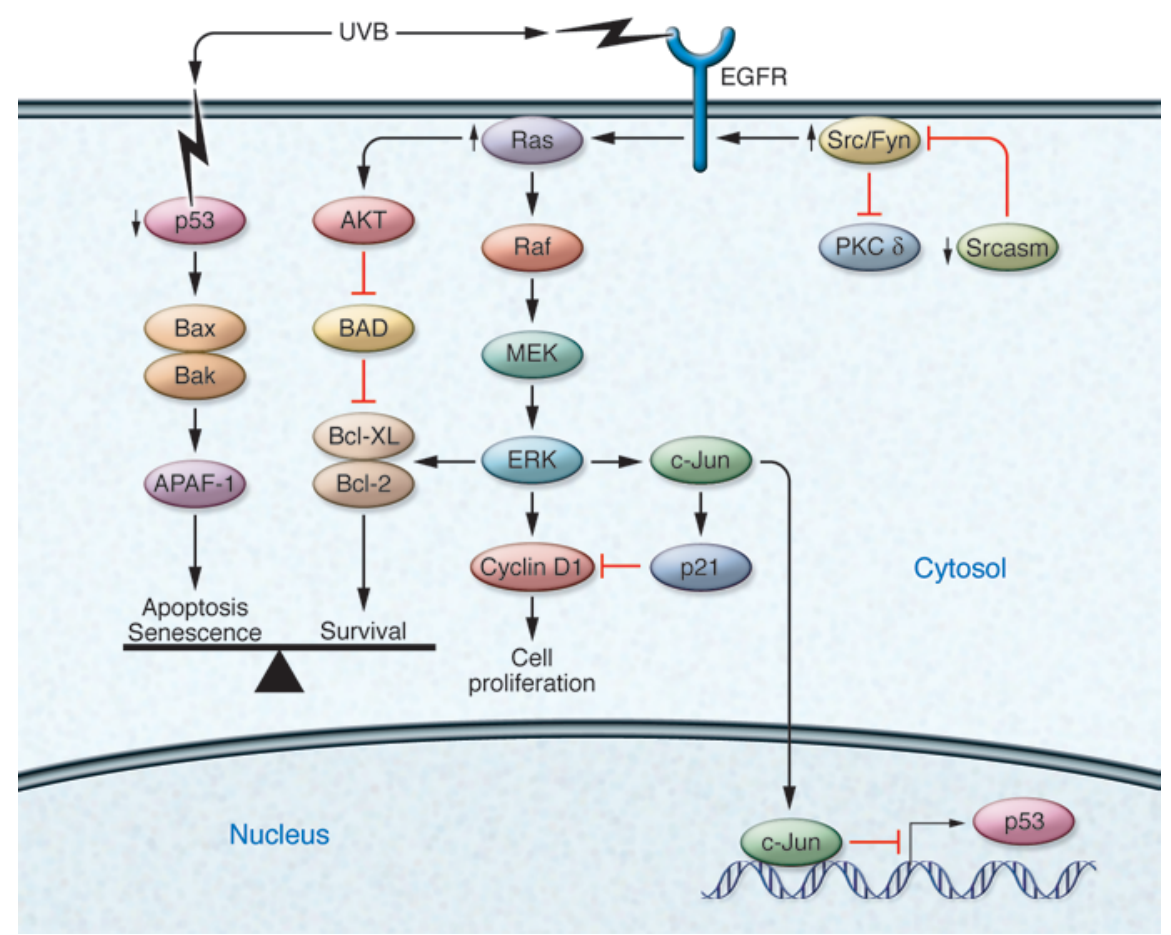

Figure 3

Key signaling pathways involved in the formation of CSCC. Mutations induced by UVB exposure can perturb multiple cellular pathways leading contribute to the formation of cSCC. Thick arrows signify an increase or decrease of signaling in SCC. Red T-bars indicate inhibitory relationships.
Ras (61). In these studies, oncogenic Hras dramatically induced Fyn mRNA but not the related kinases Src or Yes (61). Fyn was required for cell invasion and motility, and Fyn also was necessary for Hrasinduced activation of focal adhesion kinase (61). Hras upregulates Fyn mRNA through an Akt-dependent mechanism, and since Fyn activates the PI3K/Akt pathway, it may amplify its own expression through a positive feedback mechanism (29). These data suggest an interesting biological relationship between Ras and Fyn in cutaneous neoplasia that should be further evaluated and could be explored through topical application of small molecule kinase inhibitors (SMKIs), which are used to treat systemic cancers and have the physical properties required to penetrate skin. The ideal SMKIs would target Fyn and related tyrosine kinases or would target kinases in the Ras pathway. Inhibition of these signaling nodes could manifest chemoablative properties on AKs and cSCCs. One potential candidate is dasatinib, which is smaller than 500 daltons and targets multiple tyrosine kinases, including Fyn (62).

SFKs, including Fyn, are regulators of cell proliferation, invasion, and metastasis and have been shown to play a role in the pathogenesis of $\operatorname{cSCC}(29,61,63-65)$. Src-activating and signaling molecule (Srcasm) is a substrate and negative regulator of SFKs $(66,67)$. Srcasm has been shown to limit keratinocyte proliferation, promote differentiation, and negatively regulate EGFR and SFK signaling in primary human keratinocytes $(68,69)$. In human AKs and cSCCs, immunohistochemical studies have demonstrated that Srcasm levels are reduced compared with adjacent nonlesional epidermis $(44,68)$. The potency of Fyn in promoting skin tumorigenesis was confirmed in the phenotype of K14Fyn Y528F mice (described below), which spontaneously develop precancerous lesions resembling AKs and CSCCs at five to eight weeks of age (29). Analysis of these lesions revealed activation of three pro-oncogenic signaling pathways: PDK-1/Akt/mTOR, MEK/ERK, and STAT3. Activation of these signaling pathways coupled with Fyn-induced downregulation of p53 and Notch1 represents a strong oncogenic signal that can induce spontaneous skin tumor formation even in the C57BL/ 6 tumor-resistant genetic background (29). Increasing Srcasm levels with a K14-Srcasm transgene completely inhibits Fyn-induced skin neoplasia. The relevance of these in vivo murine observations to human disease was demonstrated by the finding that human cSCCs demonstrate elevated Fyn and activated SFK levels coupled with decreased Srcasm levels when compared with adjacent nonlesional skin (29). These data support the hypothesis that Srcasm functions as a tumor suppressor in human cSCCs and that inhibiting SFKs or enhancing Srcasm function may be a potential therapeutic strategy for treating cutaneous neoplasia.

Recent work has shown that PKC- $\delta$ mRNA is markedly downregulated in human cSCCs compared to epidermis suggesting that decreased PKC- $\delta$ levels are important for UVB-induced neoplasia (70). Fyn phosphorylates PKC- $\delta$, and PKC- $\delta$ is an important regulator of UVB-induced apoptsis; it will be important to determine how Fyn and Srcasm regulate PKC- $\delta$ phosphorylation and protein/ mRNA levels and correlate this UVB-induced apoptosis $(71,72)$.

Notch signaling has been implicated in both normal epidermal development and in the pathogenesis of CSCC (73). Notch signaling regulates many important cellular processes including stem cell maintenance, cell fate decisions, differentiation, proliferation, and apoptosis (74). There are four mammalian Notch proteins; Notch 1 is expressed in all the layers of the human epidermis, while Notch2 is expressed in the basal layer (73). Notch signaling promotes keratinocyte differentiation by several mechanisms including the induction of the cell cycle inhibitor p21, and was shown to decrease proliferation and increase the differentiation of embryonic keratinocytes through the activation of caspase- 3 and PKC- $\delta$ (75). In a panel of surgically excised human cSCCs, Notch 1 expression was reduced in comparison with nonlesional epidermal controls (76). Notch1 is a direct transcriptional target of p53 in human keratinocytes (76), and given the frequency of 


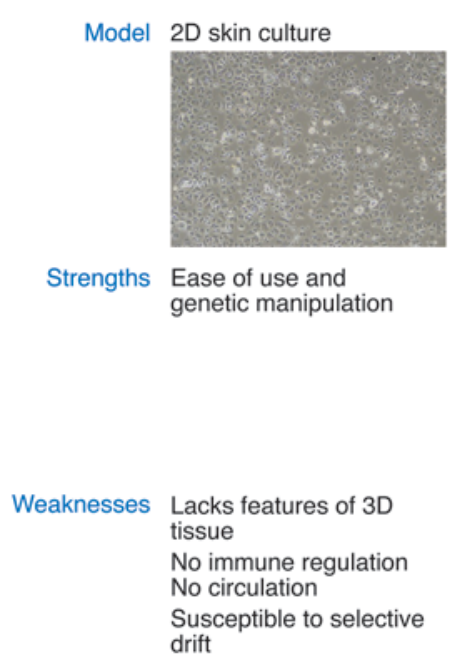

Organotypic skin culture

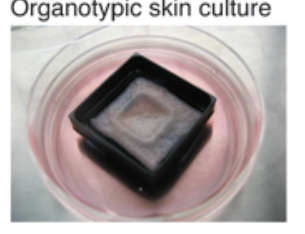

Direct genetic manipulation with lentiviruses

Architecturally faithfu native $3 \mathrm{D}$ matrix and stroma

Replication of cell-ECM interaction and invasion

No immune regulation No circulation, diffusion limited

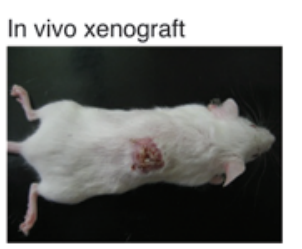

Only method for studying genetically engineered functional human tissue in $3 \mathrm{D}$ in vivo

Other strengths the same as organotypic culture

No immune regulation Murine host biology

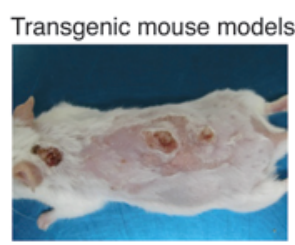

Direct genetic engineering Ability to analyze multiple genes

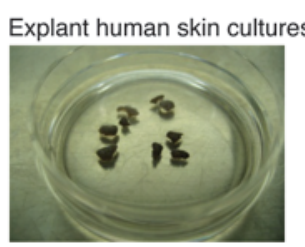

Complete human skin
Murine skin differs from human skin
Can be used for only short time periods Uniform genetic manipulation difficult

\section{Figure 4}

Comparative analysis of models used to study CSCC. The commonly used models for studying cSCC are listed with corresponding strengths and weaknesses.

p53 mutation as an early event in the pathogenesis of AKs and cSCCs, Notch1 downregulation may be a downstream consequence of p53 alteration in these lesions. Notch1 is downregulated by EGFR and Fyn through inhibition of $p 53$ transcription, in a mechanism that also involves activation of c-Jun $(28,29)$. Therefore, the oncogenic consequence of EGFR and Fyn activation includes the downregulation of p53 and Notch1. Acting as a keratinocyte tumor suppressor, Notch1 was shown to repress the tumorigenic Wnt and the Sonic hedgehog (Shh) signaling pathways in Notch1-deficient mice $(74,77)$. Loss of Notch1 signaling in primary human keratinocytes and murine epidermis results in aberrant activation of $\beta$-catenin, an effector of Wnt signaling. Conversely, expression of a dominant active form of Notch 1 in primary keratinocytes represses $\beta$-catenin-mediated transcriptional activation and depressed $\beta$-catenin protein levels (77). Notch 1 expression in the epidermis prevents the induction of SCC and BCC-like lesions in murine topical chemical carcinogenesis models. (77). Notch deficiency may inhibit DNA repair mechanisms and pro-apoptotic pathways, rendering cells more sensitive to chemical mutagens (77). Indeed, injection of nude mice with Notch1-deficient primary keratinocytes expressing activated Ras or with keratinocytes expressing activated Ras treated with a pharmacological inhibitor of Notch signaling results in the formation of poorly differentiated, highly invasive SCCs (76). These results suggest that pro-oncogenic mutations in the setting of Notch1 deficiency may enhance the malignant potential of tumors.

STAT3 belongs to a family of transcription factors that modulate a variety of target genes involved in apoptosis, angiogenesis, and cell cycle regulation. The role of STAT3 in UVB-induced cutaneous tumorigenesis was evaluated using transgenic and gene-deficient mouse models with constitutively active STAT3 expressed in the skin (K5-Stat3C) or skin-specific STAT3 deficiency (78). Following UVB exposure, the K5-Stat3C mice developed cSCCs at a greater frequency and with an increased multiplicity than control mice (78). Conversely, epidermal STAT3 deficiency protected mice from UVB tumorigenesis. The K5-STAT3 mouse epidermis had an elevated expression of antiapoptotic mediator Bcl-xL, and consequently the keratinocytes in this model were resistant to UVBinduced apoptosis (47). A diagram of the key signaling events that promote cSCC formation is shown in Figure 3.

\section{Mouse models of SCC}

Understanding human neoplasia relies on, but also has been limited by, the experimental models used to study tumor initiation and progression. Transgenic and "knockout" mice have proven to be useful in vivo models to study epidermal tumorigenesis. Transgenic expression of ErbB2, Src, Fyn, and MEK-1 kinases driven by keratin promoters in the epidermis can spontaneously produce cutaneous neoplasia leading to cSCCs $(29,64,65,79)$. K14-Fyn Y528F transgenic mice, which express an activated form of Fyn in the epidermis, produce flat precancerous lesions resembling human AKs rather than papillomas, which are seen in the classic "two-step" chemical carcinogenesis models but are not seen in human UVB-induced cutaneous neoplasia (29). These studies suggest that upregulation of the EGFR/Fyn/Src/Erk pathway appears critical for promoting cSCC formation. In support of this hypothesis, K14-ER:Ras transgenic mice, which harbor a tamoxifen-inducible Hras G12V, demonstrate a Ras-dependent epidermal hyperplasia associated with impaired differentiation resembling SCCIS (80). Other transgenic mouse lines with increased STAT3 activity also demonstrate spontaneous and UVB-induced cutaneous neoplasia leading to cSCCs (78).

Although transgenic mice are a strong in vivo model for studying cSCC formation, the inherent differences in skin structure between mice and humans limits direct correlation of murine models with human disease (Figure 4). Furthermore, there are differences in the cell signaling and functional profiles of oncogenes and tumor suppressor genes between mice and humans. For example, a component of the transforming actions of Ras are mediated through Ral activation in human cells, whereas they are mediated by Raf in murine fibroblasts (81). Therefore, correlation of experimental observations made in murine models with human tissue is key for demonstrating relevance to the human disease. 


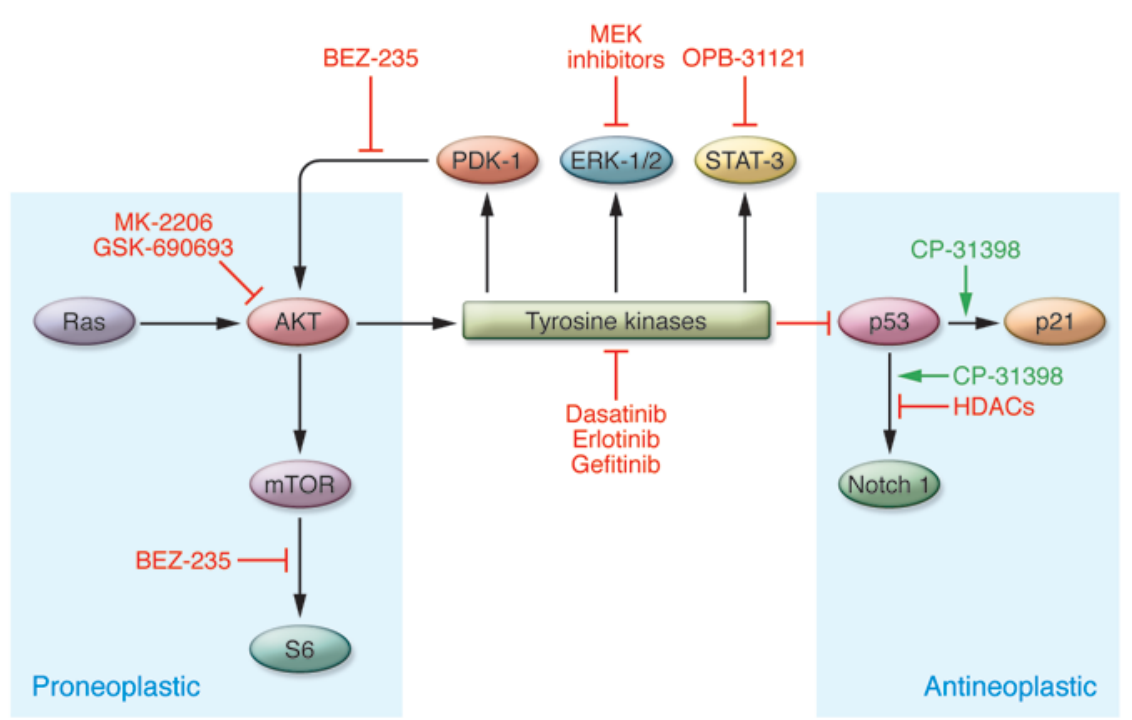

\begin{abstract}
Figure 5
Potential pathways that may be targeted by small molecules to treat AKs and cSCCs. Key signaling pathways driving cutaneous neoplasia are shown. Small molecules that can permeate the epidermis and target these signaling deficits may have therapeutic potential. Arrows denote stimulatory relationships and T-bars denote inhibitory relationships.
\end{abstract}

\section{Noncoding RNAs in SCC}

In addition to mutations in classic protein-coding genes, development and progression of SCC is likely influenced by alterations in specific noncoding RNA transcripts, including short, similarly structured, approximately 22-nucleotide miRNAs and long, variably structured (200-nt to $100-\mathrm{kb})$ lncRNAs. Because this field is in its relative infancy, functional mechanistic studies pertaining to these molecules are lacking in keratinocyte neoplasias. However, given their emerging importance as factors regulating both centrally acting cancer-associated pathways such as p53 in other tissues (82) and stem cell maintenance pathways in epidermis (83), it is likely that they will also serve similar functions in cSCC.

Consistent with a likely role of miRNA in cSCC development, SCC tumor tissue is associated with increased activity of Drosha, a key element required for miRNA maturation (84). Further, the miRNA profile in epidermis is altered in SCC, with both tumors and UVA-exposed keratinocytes displaying elevated levels of miR21 (85). Strong evidence supports a role for miR21 as an oncomir that suppresses tumor suppressor genes in epithelial cancers $(86,87)$. SCC tumors also display lower levels of miR203 (85), an antagonist of the p63 transcription factor required for the proliferation and maintenance of epidermal stem cells and upregulated in SCC.

MdM2 is a negative regulator of p53, and the SNP309 G allele of $M d M 2$ is associated with a mildly increased risk of developing cSCC (88). The p53 chemotherapeutic DNA damage response includes induction of lncRNAs such as p21 associated ncRNA DNA damage activated (PANDA) that regulate p53-mediated transcription of pro-apoptotic genes such as FAS (89). However, it is not yet clear whether PANDA or other lncRNAs contribute to the p53-mediated UVB DNA damage response. There are currently no studies associating or establishing functional roles for lncRNAs in cSCC, although recent evidence does suggest that at least one such transcript whose expression is associated with worse prognosis (HOTAIR) increases the invasiveness of epithelial breast cancer cells (90). As over $90 \%$ of the genome is transcribed, with the vast majority of species noncoding, future work utilizing highthroughput complete transcriptome analysis may reveal specific lncRNAs associated with SCC, with some specific sequences or structural motifs playing important roles in cancer genesis.

\section{Drug-induced cSCCs}

There are a number of clinical situations in which increased cSCC formation in patients is associated with medical conditions and drug usage. Understanding the biological effects of these drugs on human skin yields novel in vivo insights into the biology of human cSCCs.

Organ transplant recipients. A particularly high-risk population for cSCC are organ transplant recipients (OTRs). While immunosuppressive therapy used by OTRs is critical to prevent allograft rejection, it may predispose patients to multiple side effects including, but not limited to, cutaneous infections and neoplasms (91). cSCC is the most common neoplasm in OTRs, and its risk is increased by 60 - to 100 -fold in this patient population in comparison with immunocompetent controls $(92,93)$. Two primary drugs used in the immunosuppression regimen of OTRs are azathioprine, a purine synthesis inhibitor, and cyclosporine, a calcineurin inhibitor. Azathioprine was shown to increase UVA light photosensitivity (94). Unlike UVB light, the energy transmitted by UVA light is not usually sufficient to cause direct DNA damage (93). However, UVA light can cause indirect DNA damage through oxidative stress, and azathioprine was shown to augment reactive oxygen species-induced DNA damage (94). Cyclosporine and other calcineurin inhibitors have also been shown promote tumor formation independent of their actions on the host immune system $(93,95,96)$. Cyclosporine treatment promotes tumor growth in mice with SCID, which lack intact cell-mediated immunity (96). In addition, a recent study demonstrated that cyclosporine may promote SCC formation in OTRs by counteracting p53-dependent cellular senescence (95). Besides pharmacological immunosuppression, other tumor-promoting elements such as UV-induced DNA damage, human papillomaviruses, and tumor-stroma interactions have also been shown to contribute to cSCC development in transplant recipients $(92,93)$.

Voriconazole is a second-generation triazole antifungal agent that is widely used for the treatment of invasive aspergillosis and candidiasis, particularly in immunocompromised patients. Since its FDA approval in 2002, chronic voriconazole therapy has been associated with an increased incidence of invasive cSCC in immunosuppressed patients including those who have undergone solid 
organ transplant or bone marrow transplant or experienced HIV infection or chronic granulomatous disease (97). A recent casecontrol study indicated that voriconazole is an independent risk factor for development of cSCC in lung transplant patients (98) The mechanism of voriconazole-induced cutaneous carcinogenesis is not known, but exposure to UV light is likely related, particularly given the drug-mediated photosensitivity exhibited by the patients (97). Voriconazole does not absorb light in the UV spectrum, but the primary metabolite, voriconazole N-oxide, can absorb UVA and UVB wavelengths. The drug is metabolized to the $\mathrm{N}$-oxide form by cytochrome p450 enzymes located in the liver and in the gut epithelium. Keratinocytes also express many of these cytochrome $\mathrm{p} 450$ enzymes, raising the possibility of keratinocyte-mediated direct voriconazole $\mathrm{N}$-oxidation (99). The signaling pathways activated by voriconazole or its $\mathrm{N}$-oxide metabolite in keratinocytes are under investigation. Regular skin examinations and liberal use of sunscreens are recommended for patients taking voriconazole chronically.

Therapeutic modalities and insights. Current treatments for AKs involve topical and destructive modalities. The most commonly used topical agents to treat AKs are 5-FU, imiquimod (Aldara), and diclofenac (Solaraze). 5-FU is a chemotherapeutic agent that inhibits both RNA and DNA synthesis and targets dividing cells. AKs have a higher proportion of dividing cells than adjacent epidermis (100, 101). 5-FU has significant side effects, including prominent inflammation, ulceration, and even scarring; use of 5-FU is associated with a demonstrably lower quality of life for patients (102), and thus has many negative features as a first-line topical agent.

Imiquimod, a TLR7 agonist, acts through an immune-mediated mechanism that produces prominent inflammation leading to elimination of AKs. This inflammation resembles psoriasis in its intensity and at the molecular level, and has been associated with induction of autoimmune reactions such as alopecia areata and vitiligo (103). Another common topical agent to treat AKs is diclofenac, a topical non-steroidal anti-inflammatory compound with an unclear mechanism of action on AKs. Recent studies using diclofenac and imiquimod showed therapeutic weaknesses associated with poor clearance rates and significant irritation (104). Overall, the most common topical agents used to treat AKs have significant negative features, including irritation, decreased quality of life, and limited efficacy. These data indicate a potential benefit to developing topical treatments that target pathophysiology of AKs without inducing skin irritation.

Recent innovations in medicine have resulted from the application of small molecules that correct signaling deficits in human neoplasia. Systemic administration of small molecules that can enhance p53 function, such as CP-31398, can inhibit UVB-induced carcinogenesis in mice (ref. 105 and Figure 5). Imatinib (Gleevec) and related compounds have been useful in treating neoplasia associated with increased tyrosine kinase activity, including chronic myelogenous leukemia and gastrointestinal stromal tumors with c-Kit mutations (refs. 106-109 and Figure 5). As described above, the systemic application of small molecules to target kinases driving neoplasia or to activate anti-oncogenic signaling pathways could be used to treat AKs and cSCC; identifying skin-permeable small molecules that target these pathways essential for lesion formation would be a first step. The murine models of skin cancer and xenografted genetically engineered human skin discussed in this Review are good screening tools for identifying potential topical agents that may be useful in treating AKs and cSCCs.

\section{Conclusions}

A number of pathophysiologic features of human cutaneous neoplasia have been elucidated using a wide range of experimental techniques. A more complete understanding of the molecular pathways that drive these cancers may yield new therapeutic insights for treating these commons lesions.

\section{Acknowledgments}

This work is supported by the NIH (National Institute of Arthritis and Musculoskeletal and Skin Diseases grant AR051380 to J.T. Seykora and grant P30-AR057257) and by the Department of Dermatology at the University of Pennsylvania.

Address correspondence to: John T. Seykora, University of Pennsylvania Medical School, 235a Clinical Research Building, 415 Curie Blvd., Philadelphia, Pennsylvania 19104, USA. Phone: 215.898.0170; Fax: 215.573.2143; E-mail: john.seykora@uphs.upenn.edu.
1. Rogers HW, et al. Incidence estimate of nonmelanoma skin cancer in the United States, 2006. Arch Dermatol. 2010;146(3):283-287.

2. Salasche SJ. Epidemiology of actinic keratoses and squamous cell carcinoma. J Am Acad Dermatol. 2000;42(1 pt 2):4-7.

3. Marks R. An overview of skin cancers. Incidence and causation. Cancer. 1995;75(2 suppl):607-612.

4. Wolff KG, Goldsmith LA, Katz SI, Gilchrest BA, Paller AS, Leffell DJ. Fitzpatrick's Dermatology in General Medicine. 7th ed. New York, New York, USA: McGraw-Hill; 2007.

5. Thompson SC, Jolley D, Marks R. Reduction of solar keratoses by regular sunscreen use. $N$ Engl J Med. 1993;329(16):1147-1151.

6. Mittelbronn MA, Mullins DL, Ramos-Caro FA, Flowers FP. Frequency of pre-existing actinic keratosis in cutaneous squamous cell carcinoma. Int $J$ Dermatol. 1998;37(9):677-681.

7. Glogau RG. The risk of progression to invasive disease. J Am Acad Dermatol. 2000;42(1 pt 2):23-24.

8. Marks R, Rennie G, Selwood TS. Malignant transformation of solar keratoses to squamous cell carcinoma. Lancet. 1988;1(8589):795-797.

9. Criscione VD, Weinstock MA, Naylor MF, Luque C, Eide MJ, Bingham SF. Actinic keratoses: Natu- ral history and risk of malignant transformation in the Veterans Affairs Topical Tretinoin Chemoprevention Trial. Cancer. 2009;115(11):2523-2530.

10. Stockfleth E. Actinic keratoses. Cancer Treat Res. 2009; 146:227-239.

11. Cockerell CJ. Histopathology of incipient intraepidermal squamous cell carcinoma ("actinic keratosis”). J Am Acad Dermatol. 2000;42(1 pt 2):11-17.

12. Aho M, Vesterinen E, Meyer B, Purola E, Paavonen J. Natural history of vaginal intraepithelial neoplasia. Cancer. 1991;68(1):195-197.

13. Fearon ER, Vogelstein B. A genetic model for colorectal tumorigenesis. Cell. 1990;61(5):759-767.

14. Dajee M, et al. NF-kappaB blockade and oncogenic Ras trigger invasive human epidermal neoplasia. Nature. 2003;421(6923):639-643.

15. Lazarov M, et al. CDK4 coexpression with Ras generates malignant human epidermal tumorigenesis. Nat Med. 2002;8(10):1105-1114.

16. Hoeijmakers JH. Genome maintenance mechanisms for preventing cancer. Nature. 2001; 411(6835):366-374.

17. Brash DE, et al. A role for sunlight in skin cancer: UVinduced p53 mutations in squamous cell carcinoma. Proc Natl Acad Sci US A. 1991;88(22):10124-10128.

18. Jiang W, Ananthaswamy HN, Muller HK, Kripke
ML. p53 protects against skin cancer induction by UV-B radiation. Oncogene. 1999;18(29):4247-4253. 19. Kubo Y, et al. p53 gene mutations in human skin cancers and precancerous lesions: comparison with immunohistochemical analysis. J Invest Dermatol. 1994;102(4):440-444.

20. Ziegler A, et al. Sunburn and p53 in the onset of skin cancer. Nature. 1994;372(6508):773-776.

21. Nelson MA, et al. Analysis of the p53 gene in human precancerous actinic keratosis lesions and squamous cell cancers. Cancer Lett. 1994;85(1):23-29.

22. Ortonne JP. From actinic keratosis to squamous cell carcinoma. BrJ Dermatol. 2002;146(suppl 61):20-23.

23. Nakazawa $H$, et al. UV and skin cancer: specific $p 53$ gene mutation in normal skin as a biologically relevant exposure measurement. Proc Natl Acad SciUS A. 1994;91(1):360-364.

24. Campbell C, Quinn AG, Ro YS, Angus B, Rees JL. p53 mutations are common and early events that precede tumor invasion in squamous cell neoplasia of the skin. J Invest Dermatol. 1993;100(6):746-748.

25. Baker SJ, et al. p53 gene mutations occur in combination with $17 \mathrm{p}$ allelic deletions as late events in colorectal tumorigenesis. Cancer Res. 1990; 50(23):7717-7722.

26. Donghi R, Longoni A, Pilotti S, Michieli P, Della 
Porta G, Pierotti MA. Gene p53 mutations are restricted to poorly differentiated and undifferentiated carcinomas of the thyroid gland. JClin Invest. 1993;91(4):1753-1760.

27. Uchida T, et al. p53 mutations and prognosis in bladder tumors. J Urol. 1995;153(4):1097-1104.

28. Kolev V, et al. EGFR signalling as a negative regulator of Notch 1 gene transcription and function in proliferating keratinocytes and cancer. Nat Cell Biol. 2008;10(8):902-911.

29. Zhao L, et al. Srcasm inhibits Fyn-induced cutaneous carcinogenesis with modulation of Notch 1 and p53. Cancer Res. 2009;69(24):9439-9447.

30. Kim MS, et al. Increased expression of activating transcription factor 3 is related to the biologic behavior of cutaneous squamous cell carcinomas. Hum Pathol. 2011;42(7):954-959.

31. Lentini M, Schepis C, Cuppari DA, Batolo D. Tenascin expression in actinic keratosis. J Cutan Pathol. 2006;33(11):716-720.

32. Toll A, et al. MYC gene numerical aberrations in actinic keratosis and cutaneous squamous cell carcinoma. BrJ Dermatol. 2009;161(5):1112-1118.

33. Toll A, et al. Epidermal growth factor receptor gene numerical aberrations are frequent events in actinic keratoses and invasive cutaneous squamous cell carcinomas. Exp Dermatol. 2010;19(2):151-153.

34. Sekulic A, et al. Loss of inositol polyphosphate 5phosphatase is an early event in development of cutaneous squamous cell carcinoma. Cancer Prev Res (Phila). 2010;3(10):1277-1283.

35. Einspahr JG, et al. Relationship of p53 mutations to epidermal cell proliferation and apoptosis in human UV-induced skin carcinogenesis. Neoplasia. 1999;1(5):468-475.

36. Rehman I, Takata M, Wu YY, Rees JL. Genetic change in actinic keratoses. Oncogene. 1996; 12(12):2483-2490.

37. Purdie KJ, et al. Allelic imbalances and microdeletions affecting the PTPRD gene in cutaneous squamous cell carcinomas detected using single nucleotide polymorphism microarray analysis. Genes Chromosomes Cancer. 2007;46(7):661-669.

38. Mortier L, et al. Progression of actinic keratosis to squamous cell carcinoma of the skin correlates with deletion of the 9p21 region encoding the p16(INK4a) tumor suppressor. Cancer Lett. 2002;176(2):205-214

39. Pacifico A, Goldberg LH, Peris K, Chimenti S, Leone G, Ananthaswamy HN. Loss of CDKN2A and p14ARF expression occurs frequently in human nonmelanoma skin cancers. BrJ Dermatol. 2008;158(2):291-297.

40. Burnworth B, et al. The multi-step process of human skin carcinogenesis: a role for p53, cyclin D1, hTERT, p16, and TSP-1. Eur J Cell Biol. 2007; 86(11-12):763-780.

41. Nindl I, et al. Low prevalence of p53, p16(INK4a) and Ha-ras tumour-specific mutations in lowgraded actinic keratosis. Br J Dermatol. 2007; 156(suppl 3):34-39.

42. Pierceall WE, Goldberg LH, Tainsky MA, Mukhopadhyay T, Ananthaswamy HN. Ras gene mutation and amplification in human nonmelanoma skin cancers. Mol Carcinog. 1991;4(3):196-202.

43. Spencer JM, Kahn SM, Jiang W, DeLeo VA, Weinstein IB. Activated ras genes occur in human actinic keratoses, premalignant precursors to squamous cell carcinomas. Arch Dermatol. 1995;131(7):796-800.

44. Boukamp P. Non-melanoma skin cancer: what drives tumor development and progression? Carcinogenesis. 2005;26(10):1657-1667.

45. Khavari PA. Modelling cancer in human skin tissue. Nat Rev Cancer. 2006;6(4):270-280.

46. Bamford S DE, et al. The COSMIC (Catalogue of Somatic Mutations in Cancer) database and website; http://www.sanger.ac.uk/cosmic. Br J Cancer. 2004;91(2):355-358.

47. Marinkovich MP. Tumour microenvironment: laminin 332 in squamous-cell carcinoma. Nat Rev Cancer. 2007;7(5):370-380.

48. Martins VL, et al. Increased invasive behaviour in cutaneous squamous cell carcinoma with loss of basement-membrane type VII collagen. J Cell Sci. 2009;122(pt 11):1788-1799.

49. Reuter JA, et al. Modeling inducible human tissue neoplasia identifies an extracellular matrix interaction network involved in cancer progression. Cancer Cell. 2009;15(6):477-488.

50. Kamstrup MR, Gniadecki R, Skovgaard GL. Putative cancer stem cells in cutaneous malignancies. Exp Dermatol. 2007;16(4):297-301.

51. Morris RJ, Coulter K, Tryson K, Steinberg SR. Evidence that cutaneous carcinogen-initiated epithelial cells from mice are quiescent rather than actively cycling. Cancer Res. 1997;57(16):3436-3443.

52. Bailleul B, et al. Skin hyperkeratosis and papilloma formation in transgenic mice expressing a ras oncogene from a suprabasal keratin promoter. Cell. 1990;62(4):697-708.

53. Brown K, Strathdee D, Bryson S, Lambie W, Balmain A. The malignant capacity of skin tumours induced by expression of a mutant $\mathrm{H}$-ras transgene depends on the cell type targeted. Curr Biol. 1998; 8(9):516-524.

54. Lapouge $G$, et al. Identifying the cellular origin of squamous skin tumors. Proc Natl Acad Sci U S A. 2011;108(18):7431-7436.

55. Brittan $\mathrm{M}$, et al. Bone marrow cells engraft within the epidermis and proliferate in vivo with no evidence of cell fusion. J Pathol. 2005;205(1):1-13.

56. Li HC, Stoicov C, Rogers AB, Houghton J. Stem cells and cancer: evidence for bone marrow stem cells in epithelial cancers. World J Gastroenterol. 2006;12(3):363-371.

57. Jin $\mathrm{H}$, et al. A homing mechanism for bone marrow-derived progenitor cell recruitment to the neovasculature. J Clin Invest. 2006;116(3):652-662.

58. Ridky TW, Chow JM, Wong DJ, Khavari PA. Invasive three-dimensional organotypic neoplasia from multiple normal human epithelia. Nat Med. 2010;16(12):1450-1455

59. Zhang JY, Tao S, Kimmel R, Khavari PA. CDK4 regulation by TNFR1 and JNK is required for NFkappaB-mediated epidermal growth control. $J$ Cell Biol. 2005;168(4):561-566.

60. van Hogerlinden M, Rozell BL, Ahrlund-Richter L, Toftgard R. Squamous cell carcinomas and increased apoptosis in skin with inhibited Rel/ nuclear factor-kappaB signaling. Cancer Res. 1999; 59(14):3299-3303.

61. Yadav V, Denning MF. Fyn is induced by Ras/PI3K/ Akt signaling and is required for enhanced invasion/migration. Mol Carcinog. 2011;50(5):346-352.

62. Muller B. Imatinib and its successors--how modern chemistry has changed drug development. Curr Pharm Des. 2009;15(2):120-133.

63. Ayli EE, Li W, Brown TT, Witkiewicz A, Elenitsas $\mathrm{R}$, Seykora JT. Activation of Src-family tyrosine kinases in hyperproliferative epidermal disorders. J Cutan Pathol. 2008;35(3):273-277.

64. Matsumoto T, et al. Overexpression of a constitutively active form of c-src in skin epidermis increases sensitivity to tumor promotion by 12-O-tetradecanoylphorbol-13-acetate. Mol Carcinog. 2002; 33(3):146-155.

65. Matsumoto T, et al. Targeted expression of c-Src in epidermal basal cells leads to enhanced skin tumor promotion, malignant progression, and metastasis. Cancer Res. 2003;63(16):4819-4828.

66. Li W, Marshall C, Mei L, Gelfand J, Seykora JT. Srcasm corrects Fyn-induced epidermal hyperplasia by kinase down-regulation. J Biol Chem. 2007;282(2):1161-1169

67. Seykora JT, Mei L, Dotto GP, Stein PL. 'Srcasm: a novel Src activating and signaling molecule. J Biol Chem. 2002;277(4):2812-2822.
68. Li W, et al. Srcasm modulates EGF and Src-kinase signaling in keratinocytes. J Biol Chem. 2005; 280(7):6036-6046.

69. Li W, Marshall C, Mei L, Gelfand J, Seykora JT. Srcasm corrects fyn-induced epidermal hyperplasia by kinase down-regulation. J Biol Chem. 2007; 282(2):1161-1169.

70. Yadav V, Yanez NC, Fenton SC, Denning MF. Loss of protein kinase $\mathrm{C}$ delta gene expression in human squamous cell carcinomas: a laser capture microdissection study. Am J Pathol. 2010;176(3):1091-1096.

71. Denning MF, Wang Y, Nickoloff BJ, Wrone-Smith T. Protein kinase C delta is activated by capasedependent proteolysis during ultraviolet radiation-induced apoptosis of human keratinocytes. J Biol Chem. 1998;273(45):29995-30002.

72. Joseloff E, et al. Src family kinases phosphorylate protein kinase $\mathrm{C}$ delta on tyrosine residues and modify the neoplastic phenotype of skin keratinocytes. J Biol Chem. 2002;277(14):12318-12323.

73. Dotto GP. Notch tumor suppressor function. Oncogene. 2008;27(38):5115-5123.

74. Okuyama R, Tagami H, Aiba S. Notch signaling: its role in epidermal homeostasis and in the pathogenesis of skin diseases. J Dermatol Sci. 2008; 49(3):187-194.

75. Okuyama R, et al. High commitment of embryonic keratinocytes to terminal differentiation through a Notch1-caspase 3 regulatory mechanism. Dev Cell. 2004;6(4):551-562.

76. Lefort $\mathrm{K}$, et al. Notch 1 is a p 53 target gene involved in human keratinocyte tumor suppression through negative regulation of ROCK1/2 and MRCKalpha kinases. Genes Dev. 2007;21(5):562-577.

77. Nicolas M, et al. Notch1 functions as a tumor suppressor in mouse skin. Nat Genet. 2003;33(3):416-421.

78. Kim DJ, Angel JM, Sano S, DiGiovanni J. Constitutive activation and targeted disruption of signal transducer and activator of transcription 3 (Stat3) in mouse epidermis reveal its critical role in UVB-induced skin carcinogenesis. Oncogene. 2009; 28(7):950-960.

79. Scholl FA, Dumesic PA, Khavari PA. Mek1 alters epidermal growth and differentiation. Cancer Res. 2004;64(17):6035-6040.

80. Tarutani M, Cai T, Dajee M, Khavari PA. Inducible activation of Ras and Raf in adult epidermis. Cancer Res. 2003;63(2):319-323.

81. Hamad NM, et al. Distinct requirements for Ras oncogenesis in human versus mouse cells. Genes Dev. 2002;16(16):2045-2057.

82. Huarte $M$, et al. A large intergenic noncoding rna induced by p 53 mediates global gene repression in the 553 response. Cell. 2010;142(3):409-419.

83. Zhang L, Stokes N, Polak L, Fuchs E. Specific microRNAs are preferentially expressed by skin stem cells to balance self-renewal and early lineage commitment. Cell Stem Cell. 2011;8(3):294-308.

84. Sand M, et al. Expression levels of the microRNA processing enzymes drosha and dicer in epithelial skin cancer. Cancer Invest. 2010;28(6):649-653.

85. Dziunycz P, et al. Squamous cell carcinoma of the skin shows a distinct microRNA profile modulated by UV radiation. J Invest Dermatol. 2010; 130(11):2686-2689.

86. Lou Y, Yang X, Wang F, Cui Z, Huang Y. MicroRNA-21 promotes the cell proliferation, invasion and migration abilities in ovarian epithelial carcinomas through inhibiting the expression of PTEN protein. Int J Mol Med. 2010;26(6):819-827.

87. Moriyama T, et al. MicroRNA-21 modulates biological functions of pancreatic cancer cells including their proliferation, invasion, and chemoresistance. Mol Cancer Ther. 2009;8(5):1067-1074.

88. Almquist LM, et al. The role of TP53 and MDM2 polymorphisms in TP53 mutagenesis and risk of non-melanoma skin cancer. Carcinogenesis. 2011; 32(3):327-330. 
89. Hung T, et al. Extensive and coordinated transcription of noncoding RNAs within cell-cycle promoters. Nat Genet. 2011;43(7):621-629.

90. Gupta RA, et al. Long non-coding RNA HOTAIR reprograms chromatin state to promote cancer metastasis. Nature. 2010;464(7291):1071-1076

91. Euvrard S, Kanitakis J, Claudy A. Skin cancers after organ transplantation. $N$ Engl J Med. 2003; 348(17):1681-1691.

92. Berg D, Otley CC. Skin cancer in organ transplant recipients: Epidemiology, pathogenesis, and management. J Am Acad Dermatol. 2002;47(1):1-17.

93. Hofbauer GF, Bouwes Bavinck JN, Euvrard S. Organ transplantation and skin cancer: basic problems and new perspectives. Exp Dermatol. 2010; 19(6):473-482.

94. O'Donovan P, et al. Azathioprine and UVA light generate mutagenic oxidative DNA damage. Science. 2005;309(5742):1871-1874.

95. Wu X, et al. Opposing roles for calcineurin and ATF3 in squamous skin cancer. Nature. 2010; 465(7296):368-372.

96. Hojo M, et al. Cyclosporine induces cancer progression by a cell-autonomous mechanism. Nature. 1999;397(6719):530-534.

97. Cowen EW, et al. Chronic phototoxicity and aggres- sive squamous cell carcinoma of the skin in children and adults during treatment with voriconazole. J Am Acad Dermatol. 2010;62(1):31-37.

98. Vadnerkar A, et al. Voriconazole exposure and geographic location are independent risk factors for squamous cell carcinoma of the skin among lung transplant recipients. J Heart Lung Transplant. 2010;29(11):1240-1244

99. Baron JM, et al. Expression of multiple cytochrome P450 enzymes and multidrug resistance-associated transport proteins in human skin keratinocytes. Invest Dermatol. 2001;116(4):541-548.

100. Carpenter PM, et al. Nuclear morphometry and molecular biomarkers of actinic keratosis, sundamaged, and nonexposed skin. Cancer Epidemiol Biomarkers Prev. 2004;13(12):1996-2002.

101.Talghini S, Halimi M, Baybordi H. Expression of P27, Ki67 and P53 in squamous cell carcinoma, actinic keratosis and Bowen disease. PakJ Biol Sci. 2009;12(12):929-933.

102. Weinstock MA, Lee KC, Chren MM, Marcolivio K. Quality of life in the actinic neoplasia syndrome: The VA Topical Tretinoin Chemoprevention (VATTC) Trial. J Am Acad Dermatol. 2009;61(2):207-215.

103.van der Fits L, et al. Imiquimod-induced psoriasislike skin inflammation in mice is mediated via the
IL-23/IL-17 axis. J Immunol. 2009;182(9):5836-5845. 104. Kose O, Koc E, Erbil AH, Caliskan E, Kurumlu Z. Comparison of the efficacy and tolerability of $3 \%$ diclofenac sodium gel and 5\% imiquimod cream in the treatment of actinic keratosis. J Dermatolog Treat. 2008;19(3):159-163.

105.Tang X, et al. CP-31398 restores mutant p53 tumor suppressor function and inhibits UVBinduced skin carcinogenesis in mice. J Clin Invest. 2007;117(12):3753-3764.

106.Druker BJ, et al. Activity of a specific inhibitor of the BCR-ABL tyrosine kinase in the blast crisis of chronic myeloid leukemia and acute lymphoblastic leukemia with the Philadelphia chromosome. NEngl J Med. 2001;344(14):1038-1042.

107. Druker BJ, et al. Efficacy and safety of a specific inhibitor of the BCR-ABL tyrosine kinase in chronic myeloid leukemia. N Engl J Med. 2001; 344(14):1031-1037.

108. Druker BJ, et al. Effects of a selective inhibitor of the Abl tyrosine kinase on the growth of Bcr-Abl positive cells. Nat Med. 1996;2(5):561-566.

109. Joensuu $\mathrm{H}$, et al. Effect of the tyrosine kinase inhibitor STI571 in a patient with a metastatic gastrointestinal stromal tumor. N Engl J Med. 2001; 344(14):1052-1056 